\title{
1.2 Diabetic cardiac autonomic neuropathy: benfotiamine, insulin resistance and some pro- and anti-inflammatory parameters
}

Diabetic autonomic neuropathy (DAN) is often underestimated and indeed a serious complication of diabetes mellitus (DM), as can potentially affect any circuit/tract of autonomic nervous system (ANS). The most studied and common form of DAN is cardiac autonomic neuropathy $(\mathrm{CAN})$, owing to its relations with other microangiopathic comorbidities and life-threatening complications. Development of CAN is characterized by lesion of nerve fibers in the parasympathetic and sympathetic divisions of the ANS $[6,7]$.

The broad term that describes any malfunction or disease of the ANS is dysautonomia. It has been proved that functional changes in the ANS are associated with various forms of mild to moderate vitamin deficiencies. Mild to moderate thiamine deficiency (TD) and/or hypoxia both give rise to exaggeration of centrally controlled mechanisms involved in all survival reflexes, mediated normally through a balanced reaction of the endocrine system and ANS [8]. Due to increased requirements deriving from amplified and accelerated glucose metabolism in non-insulin dependent tissues, DM might be considered as TD state [8,9]. The TD in clinical diabetes may increase the fragility of vascular cells to the adverse effects of hyperglycemia and there by the increase of the risk of microvascular complications $[7,8]$. Chronic exposure to moderate and severe hypoxia increases the activity of the sympathetic nervous system and adrenal medulla, and TD induces an early central muscarinic cholinergic lesions [8]. It was showed that prescription of benfotiamine, is associated with reduction of oxidative stress (OS) and diabetic micro-macrovascular complications. OS was considered as the main pathophysiological pathway of CAN development. Elevated intracellular levels of glucose lead to activation of polyol pathway, formation of advanced glycation end-products (AGEs), resulting in subsequent formation of reactive oxygen species (ROS) [9].

The correction of TD must be performed using exogenous vitamin $\mathrm{B}_{1}$, or benfotiamine (high-bioavailable liposoluble vitamin $\mathrm{B}_{1}$ derivatives). Results of experimental and clinical studies suggest a positive effect of BET prescription on 
prevention of diabetic vascular disease progression. Benfotiamine broad therapeutic potential has a good efficiency on medications containing soluble thiamine derivatives for the purpose of regulating the activity of free radical processes; correction of endothelial dysfunction in case of cardiovascular diseases (CVD), stabilization of clinical and antioxidants effects [10].

The aim of this study is to analyze the effect of benfotiamine on the insulin resistance, the content of some pro- and anti-inflammatory factors in patients with type 2 DM (T2DM) and advanced stage of CAN. Forty patients with T2DM and definite CAN, aged between 50-59 years with disease duration 1-6 years, median BMI $27,2 \pm 0,34 \mathrm{~kg} / \mathrm{m}^{2}$ and median glycated hemoglobin A1c (HbA1c) $7.16 \pm 0.19 \%$ were examined. The standard hypoglycemic treatment of DM included dietary regime, appropriate physical activity, and oral antihyperglycemic drugs. Patients with T2DM and CAN were divided into 2 groups. Patients in groups did not differ significantly in age, sex distribution, BMI, duration of the disease which made them as homogeneous as possible. First group received traditional antihyperglycemic therapy $(n=19$, control) for three months; patients in group 2 ( $n=21$, treatment group), received in addition 300 $\mathrm{mg} / \mathrm{q}$.d. benfotiamine for three months. To determine the effects of benfotiamine on the investigated parameters all measurements were performed initially and after the end of treatment period. Each patient examined before the beginning of the study did not take benfotiamine and was on stable regime of hypoglycemic and antihypertensive treatment for 6 months. Inclusion criteria: age: 50-60 years old; T2DM with optimal/suboptimal glycemic control; T2DM patients with advanced stage of CAN; clinical stages of diabetic polyneuropathy; BMI within $20-30 \mathrm{~kg} / \mathrm{m}^{2}$; consent to maintain appropriate physical activity.

T2DM was diagnosed according to [11], and CAN - [6]. All patients underwent screening for CAN that included five cardiovascular autonomic reflex tests (CARTs). Resting 12-lead surface ECG and Holter-ECG [(ECG "EC-3H” ("Labtech,” Hungary)] analysis included measurement of 24 hours ECG, circadian indexes and heart rate variability parameters. The severity of CAN was determined on the condition that all five CARTs were performed. The results were considered according to scores obtained 
in individual tests. Physiological values were evaluated as " 0 " scores, borderline values - as "0.5" scores, pathological values - as " 1 " score [6]. The scores were summed up and the median score for studied patients was $2.8 \pm 0.29$.

The concentration of glucose in the blood was determined by the glucose oxidase method while HbA1c was assessed using a highly sensitive method of ion-exchange liquid chromatography with D-10 analyzer and BIO-RAD reagents (USA). Determination of immunoreactive insulin (IRI) was performed using commercial kits from immunogen insulin immunoradiometric assay reagents (Czech Republic); leptin level-from Immunotech Leptin (Czech Republic) test kits; tumor necrosis factor-alpha (TNF-alpha), interleukin (IL)-6, IL-8 and IL-10-from Vector-Best (Russia); highsensitivity C-reactive protein (hs-CRP) - from diagnosis-related group (USA). TNFalpha/IL-10 ratio and homeostasis model assessment (HOMA) insulin resistance (IR) index (HOMA-IR) were calculated. The work was done according to the principles of the Declaration of Helsinki (2004). All patients signed an informed consent prior their inclusion in the study.

The normality of presented data were checked by using Shapiro-Wilk test, all the studied variables had a normal distribution. Absolute values were compared with calculation of mean values, errors of means, using Student's t-test. Data are presented as mean \pm standard error of the mean ( $\Delta \%, \mathrm{Mean} \pm \mathrm{SEM})$. Statistical significance was set at $\mathrm{p}<0.05$. All tests were performed using the ANOVA (MicroCal Origin v. 8.0) software.

\section{Results}

The level of HbA1c in patients with T2DM and definite CAN did not change significantly after treatment $(p>0.05)$. Changes of glucose, IRI concentrations and HOMA-IR parameters in patients with T2DM and definite CAN after 3-months of benfotiamine therapy are given in table 1 . 
Table 1

The glucose, IRI concentrations and HOMA-IR parameters after 3-months of benfotiamine therapy $(\Delta \%$, Mean \pm SEM)

\begin{tabular}{|c|c|c|c|c|c|}
\hline \multirow[t]{2}{*}{ Parameter } & \multicolumn{5}{|c|}{ Patients with T2DM and definite CAN $(\mathrm{n}=40)$} \\
\hline & Groups & $\begin{array}{l}\text { Before } \\
\text { treatment }\end{array}$ & $\begin{array}{l}\text { After } \\
\text { treatment }\end{array}$ & $\%$ change & $\mathrm{p}$ \\
\hline $\begin{array}{l}\text { Fasting } \\
\text { blood }\end{array}$ & $\begin{array}{l}\text { Control } \\
(n=19)\end{array}$ & $7.03 \pm 0.26$ & $7.05 \pm 0.30$ & $+0.89 \% \pm 3.1 \%$ & $>0.05$ \\
\hline $\begin{array}{l}\text { glucose } \\
(\mathrm{mmol} / \mathrm{L})\end{array}$ & $\begin{array}{l}\text { Treatment } \\
(\mathrm{n}=21)\end{array}$ & $6.64 \pm 0.42$ & $6.69 \pm 0.38$ & $+2.19 \% \pm 2.96 \%$ & $>0.05$ \\
\hline \multirow[t]{2}{*}{$\begin{array}{l}\text { Fasting IRI } \\
(\mu \mathrm{IU} / \mathrm{mL})\end{array}$} & $\begin{array}{l}\text { Control } \\
(n=19)\end{array}$ & $27.76 \pm 2.11$ & $26.01 \pm 2.19$ & $-6.7 \% \pm 2.0 \%$ & $>0.05$ \\
\hline & $\begin{array}{l}\text { Treatment } \\
(\mathrm{n}=21)\end{array}$ & $26.84 \pm 1.39$ & $23.21 \pm 0.86$ & $\begin{array}{c}- \\
12.74 \% \pm 1.42 \%\end{array}$ & $<0.05$ \\
\hline \multirow[t]{2}{*}{ HOMA-IR } & $\begin{array}{l}\text { Control } \\
(n=19)\end{array}$ & $9.03 \pm 0.98$ & $8.39 \pm 0.91$ & $-7.15 \% \pm 1.8 \%$ & $>0.05$ \\
\hline & $\begin{array}{l}\text { Treatment } \\
(\mathrm{n}=21)\end{array}$ & $8.06 \pm 0.89$ & $6.99 \pm 0.6$ & $\begin{array}{c}- \\
11.91 \% \pm 2.68 \%\end{array}$ & $>0.05$ \\
\hline
\end{tabular}

The results are presented as absolute values and as $\%$ change from baseline. $\Delta \%$, Mean \pm SEM; $p<0.05$, compared to baseline.

As can be seen from table 1 prescription of benfotiamine to patients with T2DM and definite CAN contributes to a statistically significant reduction in IRI levels [ $\Delta=-$ $12.74 \% \pm 1.42 \%(\mathrm{p}<0.05)]$. At the same time, prescription of benfotiamine does not affect the concentration of fasting glucose and HOMA-IR parameters ( $>0.05)$. Investigated parameters did not change significantly in the control group $(\mathrm{p}>0.05)$.

Changes of hs-CRP, leptin and TNF-alpha concentrations in blood of patients with T2DM and CAN after 3-months of benfotiamine therapy are given in table 2. 
Table 2

Changes of the hs-CRP, leptin and TNF-alpha concentrations after 3-months of benfotiamine therapy $(\Delta \%$, Mean \pm SEM)

\begin{tabular}{llllll}
\hline Parameter & \multicolumn{5}{l}{ Patients with T2DM and definite CAN (n=40) } \\
\cline { 2 - 6 } & Groups & $\begin{array}{l}\text { Before } \\
\text { treatment }\end{array}$ & $\begin{array}{l}\text { After } \\
\text { treatment }\end{array}$ & \% change & $\mathrm{p}$ \\
\hline hs-CRP & Control & $2.88 \pm 0.48$ & $2.69 \pm 0.48$ & $-7.2 \% \pm 1.64 \%$ & $>0.05$ \\
$(\mathrm{mg} / \mathrm{L})$ & $(\mathrm{n}=19)$ & & & & \\
& Treatment & $2.91 \pm 0.19$ & $2.48 \pm 0.1$ & - & $<0.05$ \\
& $(\mathrm{n}=21)$ & & & $13.62 \% \pm 1.96 \%$ & \\
Leptin & Control & $21.06 \pm 1.69$ & $19.52 \pm 1.58$ & $-7.1 \% \pm 1.81 \%$ & $>0.05$ \\
$(\mu \mathrm{g} / \mathrm{L})$ & $(\mathrm{n}=19)$ & & & & \\
& Treatment & $20.87 \pm 1.85$ & $19.61 \pm 1.84$ & $-6.41 \% \pm 1.33 \%$ & $>0.05$ \\
& $(\mathrm{n}=21)$ & & & & $>0.05$ \\
TNF-alpha & Control & $5.82 \pm 0.44$ & $5.49 \pm 0.42$ & $-6.1 \% \pm 1.02 \%$ & \\
$(\mathrm{pg} / \mathrm{mL})$ & $(\mathrm{n}=19)$ & & & & $<0.05$ \\
& Treatment & $5.38 \pm 0.2$ & $4.75 \pm 0.13$ & - & \\
& $(\mathrm{n}=21)$ & & & $10.24 \% \pm 1.54 \%$ & \\
\hline
\end{tabular}

The results are presented as absolute values and as $\%$ change from baseline. $\Delta \%$, Mean \pm SEM; $p<0.05$, compared to baseline.

It is shown in table 2 that the outcome of treatment group was better than of control group. In particular, benfotiamine prescription to patients with T2DM and definite CAN contributes to a statistically significant reduction in hs-CRP [ $\Delta=-$ $13.62 \% \pm 1.96 \% \quad(\mathrm{p}<0.05)]$ and TNF-alpha $[\Delta=-10.24 \% \pm 1.54 \% \quad(\mathrm{p}<0.05)]$ levels compared to the control group. Prescription of benfotiamine does not affect on the concentration of leptin ( $\mathrm{p}>0.05)$.

Changes of some IL's concentrations and TNF-alpha/IL-10 ratio of patients with T2DM and definite CAN after 3-months of benfotiamine therapy are given in table 3 . 
Table 3

IL-6, IL-8, IL-10 concentrations and TNF-alpha/IL-10 ratio after 3-months of benfotiamine therapy $(\Delta \%$, Mean \pm SEM)

\begin{tabular}{|c|c|c|c|c|c|}
\hline \multirow[t]{2}{*}{ Parameter } & \multicolumn{5}{|c|}{ Patients with T2DM and definite CAN $(n=40)$} \\
\hline & Groups & $\begin{array}{l}\text { Before } \\
\text { treatment }\end{array}$ & $\begin{array}{l}\text { After } \\
\text { treatment }\end{array}$ & $\%$ change & $\mathrm{p}$ \\
\hline \multirow[t]{2}{*}{$\begin{array}{l}\text { IL-6 } \\
\text { (pg/mL) }\end{array}$} & $\begin{array}{l}\text { Control } \\
(\mathrm{n}=19)\end{array}$ & $5.54 \pm 0.52$ & $5.14 \pm 0.44$ & $-5.81 \% \pm 1.79 \%$ & $>0.05$ \\
\hline & $\begin{array}{l}\text { Treatment } \\
(\mathrm{n}=21)\end{array}$ & $5.74 \pm 0.31$ & $4.79 \pm 0.22$ & $\begin{array}{c}- \\
15.41 \% \pm 2.03 \%\end{array}$ & $<0.05$ \\
\hline \multirow[t]{2}{*}{$\begin{array}{l}\text { IL-8 } \\
(\mathrm{pg} / \mathrm{mL})\end{array}$} & $\begin{array}{l}\text { Control } \\
(n=19)\end{array}$ & $7.26 \pm 0.49$ & $6.94 \pm 0.48$ & $-3.9 \% \pm 1.59 \%$ & $>0.05$ \\
\hline & $\begin{array}{l}\text { Treatment } \\
(\mathrm{n}=21)\end{array}$ & $7.12 \pm 0.48$ & $6.64 \pm 0.43$ & $-6.32 \% \pm 2.03 \%$ & $>0.05$ \\
\hline \multirow[t]{2}{*}{$\begin{array}{l}\text { IL-10 } \\
(\mathrm{pg} / \mathrm{mL})\end{array}$} & $\begin{array}{l}\text { Control } \\
(n=19)\end{array}$ & $16.73 \pm 2.53$ & $15.66 \pm 2.28$ & $-3.7 \% \pm 2.33 \%$ & $>0.05$ \\
\hline & $\begin{array}{l}\text { Treatment } \\
(\mathrm{n}=21)\end{array}$ & $16.17 \pm 1.86$ & $14.78 \pm 1.49$ & $-6.19 \% \pm 2.73 \%$ & $>0.05$ \\
\hline $\begin{array}{l}\text { TNF- } \\
\text { alpha/IL- }\end{array}$ & $\begin{array}{l}\text { Control } \\
(n=19)\end{array}$ & $42.51 \pm 5.49$ & $41.5 \pm 4.79$ & $-0.51 \% \pm 2.32 \%$ & $>0.05$ \\
\hline $10(\%)$ & $\begin{array}{l}\text { Treatment } \\
(\mathrm{n}=21)\end{array}$ & $38.61 \pm 4.27$ & $35.91 \pm 3.33$ & $-4.34 \% \pm 2.77 \%$ & $>0.05$ \\
\hline
\end{tabular}

The results are presented as absolute values and as $\%$ change from baseline. $\Delta \%$. Mean \pm SEM; $p<0.05$. compared to baseline.

As can be seen from table 3 prescription of benfotiamine to patients with T2DM and definite $\mathrm{CAN}$ is accompanied by the statistically significant decrease in the content of IL-6 $[\Delta=-15,41 \% \pm 2,03 \%(\mathrm{p}<0.05)]$, and, at the same time, does not affect on the concentration of IL-8 (p<0.05), IL-10 (p>0.05) and TNF-alpha/IL-10 ratio parameters 
( $>$ >0.05). In the control group no positive dynamics of the concentration of the studied parameters was found ( $\mathrm{p}>0.05)$.

The protective effect of high-dose thiamine on detrusor contractility and on progression of diabetic cystopathy in streptozotocin-diabetic rats was some of the findings directed to the effect of thiamine on DAN. The deficiency of thiamine in clinical DM may increase the fragility of vascular cells to the adverse effects of hyperglycemia and there by the increase of the risk of developing microvascular complications. A suppression of transketolase (TKT) activity, and subsequent downregulation of the hexose monophosphate (HMP) shunt, resulting in accumulation of glyceraldehyde 3-phosphate, fructose 6-phosphate, and dihydroxyacetone phosphate may be at least one mechanism in the development of diabetes-induced vascular damage and other comorbidities [12, 13].

Thiamine and its derivatives have been demonstrated to prevent the activation of the biochemical pathways [increased flux through the polyol pathway, formation of AGEs, activation of protein kinase $\mathrm{C}$ (PKC), and increased flux through the hexosamine biosynthesis pathway (HBP)] induced by hyperglycemia in DM. TD plays a role in the diabetic endothelial vascular diseases, such as, neuropathy [13, 14]. In vitro studies with benfotiamine have shown a reduction in PKC activation in the glomeruli and decreased glomerular AGEs levels. Benfotiamine has been shown to prevent increased markers of HBP activity, intracellular AGEs formation, intracellular PKC activity and nuclear factor kappa-light-chain-enhancer of activated B cells (NF$\kappa \mathrm{B})$ activation seen with in vitro hyperglycemic damage. High-dose therapy of thiamine and benfotiamine suppressed AGEs accumulation in the peripheral nerve and reversed diabetic neuropathies (DN's) potentially by reducing the levels of triose phosphates via activation of TKT [14].

Cardiac OS is involved in heart failure that is induced by thiamine deprivation in rats. These findings suggest that thiamine modulates OS $[14,15]$. Endothelial nitric oxide synthase (eNOS) and nitric oxide (NO) may play an important role in attenuating cardiac remodeling and apoptosis. BFT reduces OS and activates eNOS to enhance the generation and bioavailability of NO, and it subsequently improves the integrity of 
vascular endothelium to prevent sodium arsenite-induced experimental vascular endothelial dysfunction [15].

The nerve tissues in DM undergo a proinflammatory process that presents symptoms and develops DN. The levels of CRP and TNF-alpha correlate with the incidence of DN's. Production of the initiating inflammatory mediators such as TNFalpha, transforming growth factor-beta, and NF- $\kappa \mathrm{B}$ results from several glucoseinduced pathways. Cyclooxygenase- 2 (COX-2) is an important enzyme that is upregulated by NF- $\kappa \mathrm{B}$ in diabetic peripheral nerves and consecutively generates prostaglandin E2 and ROS that trigger NF- $\kappa$ B. Inducible nitric oxide synthase (iNOS) is an additional inflammatory enzyme which is regulated by NF- $\kappa \mathrm{B}$. Similar to COX2 , iNOS either induces NF- $\kappa \mathrm{B}$ or is induced by it. This gives the impression that chronic NF- $\kappa \mathrm{B}$ activation is in the center of all the inflammatory elements operating in DN's. The cytokines which are induced by NF- $\kappa$ B in Schwann cells, endothelial cells, and neurons lead to absorption of macrophages in the diabetic nerves. Macrophages promote DN's via a variety of mechanisms, including making of cytokines, ROS, and proteases, which all result in cellular oxidative damage and myelin breakdown [15, $16]$.

Benfotiamine can promote neuronal and vascular deficiency correction through participation of NO processes, which have a significant therapeutic potential for the treatment of CVD. Benfotiamine significantly decreased production of proinflammatory mediators such as inducible form of iNOS and NO; COX-2, heat-shock protein 70, TNF-alpha, IL-6, whereas it increased anti-inflammatory IL-10 production in lipopolysaccharide (LPS)-stimulated BV-2 microglia. Moreover, benfotiamine suppressed the phosphorylation of extracellular signal-regulated kinases 1/2 (ERK1/2), c-Jun N-terminal kinases (JNK) and a serine/threonine protein kinase (Akt/PKB). Treatment with specific inhibitors revealed that benfotiamine-mediated suppression of NO production was via JNK1/2 and Akt pathway, while the cytokine suppression includes ERK1/2, JNK1/2 and Akt pathways. In murine macrophages, benfotiamine also blocked the expression of COX-2 and its product, prostaglandin E2, by LPSinduces cytotoxicity. In addition, benfotiamine significantly prevented LPS-induced 
macrophage death and monocyte adhesion to endothelial cells. These antiinflammatory effects of benfotiamine are mediated through the regulation of the arachidonic acid pathway in macrophages. Therefore, benfotiamine may have therapeutic potential for neurological diseases by inhibiting inflammatory mediators and enhancing anti-inflammatory factor production $[12,17]$.

The mechanism of benfotiamine influence on diabetic CAN pathogenesis is not well-known. There is moderate evidence from preclinical experimental models that high-dose thiamine and benfotiamine (1) inhibit the HMP, AGEs formation, and diacylglycerol-PKC through the TKT activation; (2) target at various surrogate markers of hyperglycemia-induced pathological processes and (3) can delay the progression of microangiopathic complications [17]. Therefore, the positive influences of benfotiamine is partly confirmed by its neurotropic, cardioprotective, angioprotective and cytoprotective properties [18, 19]; suggests the feasibility of its usage in the complex treatment of patients with T2DM and definite CAN. The results obtained in our study indicate that benfotiamine contributed to a decrease in the hs-CRP, TNFalpha and IL-6 levels. Obtained results could witness, that prescription of benfotiamine may lead to decrease of the proinflammatory immune response. The obtained data suggest that prescription of benfotiamine is associated with reduction of proinflammatory component of the immune response and make it possible to regard benfotiamine as a promising pharmacological agent in a complex therapy of the definite stage of CAN in T2DM. Thus, further investigations aimed to understand the mechanism of action and confirmation of the beneficial effect of benfotiamine on biochemical parameters, dynamics of independent CARTs, Holter-ECG, arterial wall stiffness parameters among patients with T2DM and CAN, and its associated comorbidities may be needed to validate this clinical findings. 